\title{
Recent Advances in Wireless Communications and Networking
}

\author{
Jun Zheng • Andreas F. Molisch • Nirwan Ansari • \\ Baoxian Zhang
}

Published online: 20 February 2010

(C) Springer Science+Business Media, LLC 2010

The annual International Conference on Communications and Networking in China (CHINACOM) aims to provide a forum that brings together Chinese and international researchers and practitioners to showcase recent advances in communications networking research. CHINACOM 2008 is the third edition of this event, which was successfully held in Hangzhou, China, in August 2008. This special issue includes a collection of six outstanding research papers selected from CHINACOM 2008, which is a result of several stages of filtration and selection. First, out of the 822 submissions from 36 countries/regions in 5 continents, among which over 500 submissions were from

\section{J. Zheng $(\bowtie)$}

National Mobile Communications Research Laboratory,

Southeast University,

Nanjing, Jiangsu, China

e-mail: junzheng@seu.edu.cn

A. F. Molisch

Department of Electrical Engineering-Systems,

University of Southern California,

Los Angeles, CA, USA

e-mail: molisch@usc.edu

\section{N. Ansari}

Department of Electrical and Computer Engineering,

New Jersey Institute of Technology,

Newark, NJ, USA

e-mail: nirwan.ansari@njit.edu

\section{B. Zhang}

College of Computing and Communication Engineering, Graduate University of Chinese Academy of Sciences (GUCAS),

Beijing, China

e-mail: bxzhang@gucas.ac.cn mainland China, less than 270 papers, including some invited papers, were accepted for presentation, representing an acceptance rate of about $33 \%$. Among the 270 papers, 15 papers have been invited to extend and enhance their work for further consideration for publication in this special issue and six of them have finally been included in the special issue. We would like to use this opportunity to appreciate all the reviewers who volunteered their time and professional expertise to help us reach the selection decisions.

The papers included in this special issue cover a diversity of topics in wireless communications and networking. In the first paper, "BTAC: A Busy Tone Based Cooperative MAC Protocol for Wireless Local Area Networks", Sayed et al. proposed and analyzed a busy tone based cooperative medium access control (MAC) protocol, called BTAC, for multi-rate wireless local area networks (WLANs) based on the concept of cooperative communications. The analytical and simulation results show that the BTAC protocol is simple, robust, and fully compatible with the IEEE $802.11 \mathrm{~b}$ standard, and can achieve better throughput and delay performance than the standard distributed coordination function (DCF) protocol and the recently proposed CoopMAC protocol.

In the second paper, "Achieving End-to-end Fairness in 802.11e Based Wireless Multi-Hop Mesh Networks without Coordination," Li et al. studied the fairness issue in using 802.11 at the MAC layer and proposed the use of 802.11e's TXOP mechanism to restore/perform fair resource allocation. The proposed TXOP based mechanism is implementable on standard hardware in a simple and fully decentralized way without a need for message passing. 
In the third paper, "On the Deployment of Antenna Elements in Generalized Multi-User Distributed Antenna Systems," Feng et al. studied the impact of the deployment of antenna elements on the performance of a multi-user distributed antenna system (DAS). They proposed a generalized system model, where the antenna elements are divided into antenna clusters and the antenna clusters are randomly deployed in the coverage area, and particularly investigated the impact of the antenna cluster size on the system performance in terms of the uplink sum rate capacity.

The fourth paper, "The Maximum Throughput of a Wireless Multi-Hop Path," by Mao analytically investigates the maximum end-to-end throughput that can be achieved on a wireless multi-hop path and shows that the maximum achievable end-to-end throughput is determined by the throughput of its bottleneck. Moreover, it suggests an optimum scheduling algorithm, which can be used to achieve the maximum end-to-end throughput, and the use of the (maximal) independent sets as the basic blocks for the design of scheduling algorithms. The findings lay guidelines for the design of optimum scheduling algorithms for a wireless multi-hop network.

In the fifth paper, "Energy-efficient Tree-based Message Ferrying Routing Schemes for Wireless Sensor Networks," Zhu et al. studied and proposed a tree-based message ferrying (MF) algorithm (TMFA) with least-ratio tree (LRT) construction to reduce energy consumption in a wireless sensor network. The experimental results show that as compared to existing least-energy tree (LET) construction and minimum spanning tree (MST) construction, LRT construction can achieve a better performance in terms of the ratio of the number of packets reaching the sink to the total energy consumed by all the nodes.

In the last paper, "On Object Identification Reliability Using RFID," Wang et al. discussed object identification reliability and proposed a general framework for guaranteeing object identification reliability in RFID systems.

We would like to thank all the authors for their contributions to this special issue. We are grateful to all reviewers for their time and efforts in carefully reviewing all the papers and providing valuable review comments. We would also like to thank Katiuscia Buonaiuto and Sara Fruner, the Assistant to the Editor-In-Chief, Monaliza Castañeda, the Springer Production Editor, Paulo Francisco Montes, the Springer JEO Assistant, and all other production staff for their support during the publication process.

It is our hope that the papers included in this special issue present recent research advances in wireless communications and networking, and become an important reference for researchers and practitioners in the area. Finally, we hope that the readers will find this special issue timely and informative.

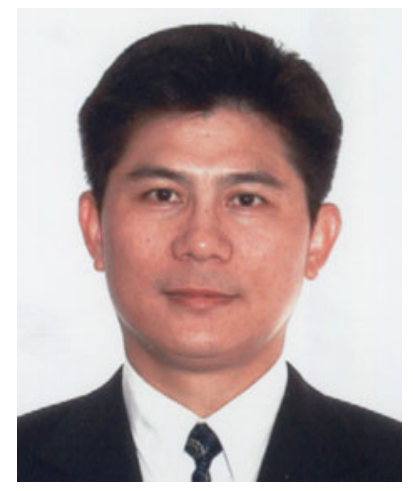

Jun Zheng (junzheng@seu. edu.cn) received the $\mathrm{Ph}$. D. degree in electrical and electronic engineering from The University of Hong Kong, Hong Kong, in 2000. He is a Full Professor at the National Mobile Communications Research Laboratory, Southeast University (SEU), Nanjing, Jiangsu, China. Before joining SEU, he was with the School of Information Technology and Engineering, University of Ottawa, Ottawa, $\mathrm{ON}$, Canada. He has co-edited (chief editor) the book Wireless Sensor Networks: A Networking Perspective (New York: Wiley-IEEE Press, 2009), and has published more than 80 technical papers in refereed journals and magazines, and peer-reviewed conference proceedings. His research interests include mobile communication networks, wireless sensor networks, and mobile ad hoc networks, focused on network architectures and protocols. Dr. Zheng serves as an Associate Technical Editor of IEEE Communications Magazine, an Editor of IEEE Communications Surveys \& Tutorials, and an editorial board member of several other refereed journals, including Elsevier Ad Hoc Networks Journal and Wiley Wireless Communications and Mobile Computing. He has coedited ten special issues for different refereed journals and magazines, including IEEE Journal on Selected Areas in Communications and IEEE Network, all as Lead Guest Editor. He has served as the founding General Chair of AdHocNets'09, General Chair of AccessNets'07, and TPC or Symposium Co-Chair for several international conferences and symposia, including IEEE GLOBECOM'08, ICC'09, GLOBECOM'10, and ICC'11. He has also served as a TPC member for a number of international conferences and symposiums. He is a senior member of the IEEE.

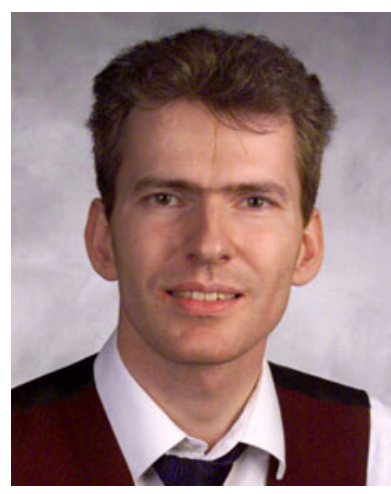

Andreas F. Molisch (molisch @usc.edu) is Professor of Electrical Engineering at the University of Southern California, Los Angeles, CA, USA. Previously, he was with AT\&T (Bell) Laboratories Research (USA), Lund University (Sweden), Mitsubishi Electric Research Labs, (USA), and TU Vienna (Austria). His current research interests are measurement and modeling of mobile radio channels, UWB, cooperative communications, and MIMO systems. He has authored, co-authored or edited four books (among them the textbook Wireless Communications, Wiley-IEEE Press), eleven book chapters, more than 120 journal papers, and numerous conference contributions, as well as more than 70 patents and 60 standards contributions. Dr. Molisch has been an editor of a number of journals and special issues, General Chair, TPC Chair, or Symposium Chair of multiple international conferences, and chairman of various international standardization groups. He is a Fellow of the IEEE, a Fellow of the IET, an IEEE Distinguished Lecturer, and recipient of several awards. 


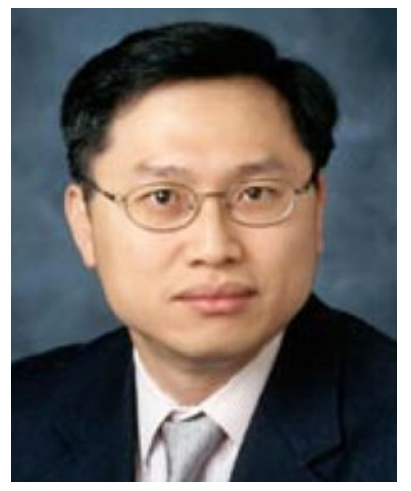

Nirwan Ansari (nirwan.ansari @ njit.edu) received the B.S.E. E. degree (summa cum laude) from the New Jersey Institute of Technology (NJIT), Newark, NJ, in 1982, the M.S.E.E. degree from the University of Michigan, Ann Arbor, MI, in 1983, and the Ph.D. degree from Purdue University, West Lafayette, IN, in 1988. He joined the Department of Electrical and Computer Engineering, NJIT, as an Assistant Professor in 1988, and has been a Full Professor since 1997. He has also assumed several administrative positions. He authored Computational Intelligence for Optimization (Kluwer, 1997) with E.S.H. Hou, and co-edited Neural Networks in Telecommunications (Kluwer, 1994) with B. Yuhas. He is a Senior Technical Editor of the IEEE Communications Magazine, and is/was also serving on the editorial/advisory board of six other journals. His current research focuses on various aspects of broadband networks and multimedia communications. He has contributed 350 technical publications, of which over one third in widely cited refereed journals and magazines. Dr. Ansari has/had been serving the IEEE in various roles such as Chair of COMSOC Technical Committee on Ad Hoc and Sensor Networks, Chair for the IEEE North Jersey Communications Society (COMSOC) Chapter, Chair of IEEE North Jersey Section, Chair of IEEE COMSOC Networking Technical Committee (TC) Cluster, a member of the IEEE Region 1 Board of Governors, and (TPC) Chair/Vice-chair of several conferences. Some of his recognitions include the 1998 NJIT Excellence Teaching Award in Graduate Instruction, a 1999 IEEE Region 1 Award, a 2007 IEEE Leadership Award, the 2008 NJIT Excellence Teaching Award in Outstanding Professional Development, the 2009 NCE Excellence in Teaching Award, the designation of IEEE Communications Society
Distinguished Lecturer (2006-2009, two terms), and an IEEE fellow (2009).

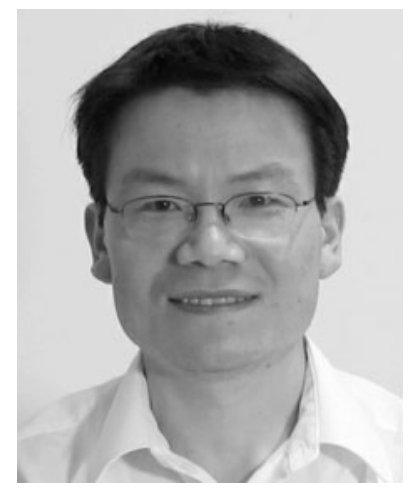

Baoxian Zhang (bxzhang@ gucas.ac.cn) is currently a Full Professor with the College of Computing and Communication Engineering of Graduate University of Chinese Academy of Sciences (GUCAS), Beijing, China. He received his BS, $\mathrm{MS}, \mathrm{PhD}$ degrees in electrical engineering from Northern Jiaotong University (Now Beijing Jiaotong University), China, in 1994, 1997, and 2000, respectively. Prior to joining GUCAS, he was a Research Scientist with School of Information Technology and Engineering (SITE) of University of Ottawa, Canada. Dr. Zhang has served as a Guest Editor for a special issue of IEEE Journal on Selected Areas in Communications on Network Coding for Wireless Communications Networks and a special issue of Wiley Wireless Communications and Mobile Computing on Underwater Wireless Sensor Networks. He has served as a Symposium Co-Chair of CHINACOM'08-Advances in Internet Symposium and IWCMC'09-Cross-Layer Optimized Wireless Networks Symposium. He has also served on the technical program committees (TPCs) for many international conferences and symposia, including IEEE GLOBECOM, ICC, and WCNC. He has published over 80 refereed technical papers in archival journals and conference proceedings. His research interests include network architecture, protocol and algorithm design, wireless ad hoc and sensor networks, and performance evaluation. He is a member of the IEEE. 